\title{
Analytical Modeling of TCP Clients in Wi-Fi Hot Spot Networks ${ }^{\star}$
}

\author{
Raffaele Bruno, Marco Conti, and Enrico Gregori \\ Italian National Research Council (CNR) - IIT Institute \\ Via G. Moruzzi, 1 - 56100 Pisa, Italy \\ \{firstname.lastname\}@iit.cnr.it
}

\begin{abstract}
The channel utilization of 802.11-based wireless local area networks has been studied extensively from an analytical perspective. However, these studies consider wireless networks formed by saturated stations that transmit UDP-like traffic to randomly selected destinations. This model cannot be easily applied to hot spots since: $i$ ) the majority of Internet applications are based on the TCP protocol; ii) the TCP traffic is rate-controlled and the TCP flow control mechanisms impede the stations to saturate; and iii) the users' traffic is either sent towards or received from the access point. This paper is the first to provide an analytical model of the channel utilization in hot spots when there are $M$ users which use TCP connections to download traffic from the Internet. Specifically, in this work we analytically investigate the complex interaction of the TCP flow control mechanisms and MAC collision avoidance techniques in $802.11 \mathrm{~b}$-based hot spot networks. We validate our model by means of simulations, and we exploit it to provide thorough reasons of the following counter-intuitive observations: $i$ ) the channel utilization is almost independent of the number of TCP connections; $i$ ) on average the access point contends with few users for the channel bandwidth.
\end{abstract}

\section{Introduction}

The IEEE $802.11 \mathrm{~b}$ standard [1], also named $W i-F i$, is the dominant technology in the market of network cards for wireless local area networks (WLANs). Now the attention of manufactures and service providers is turning to deploying WiFi-based networks over hot spots, such as cafes, retail shops, convention centers, airports and other areas where people can benefit by a seamless public access to the Internet. As the number of users increases, the throughput obtained by each user is one of the most crucial concerns for the service providers and the hot spot operators. It is important to study the MAC protocol efficiency since the 802.11b standard uses a CSMA/CA-based random access protocol that allows unconstrained movement of mobile hosts, but doesn't provide guarantees on the

\footnotetext{
* This work was carried out under the financial support of the Italian Ministry for Education and Scientific Research (MIUR) in the framework of the Projects: FIRBPERF and FIRB-VICOM
} 
stations' throughput. An extensive literature of analytical models of the channel utilization in 802.11-based networks can be found $[2,3,4,5]$. However, these studies consider wireless networks formed by saturated stations, i.e., each station has immediately a packet available for transmission, which transmit UDP-like traffic to randomly selected destinations. This model cannot be easily applied to hot spots since: $i$ ) the majority of Internet applications are based on the TCP protocol; $i i)$ the TCP traffic is rate-controlled and the TCP flow control mechanisms impede the stations to saturate; and $i i i)$ the users' traffic is either sent towards or received from the access point (AP). This paper aims at developing an analytical model that characterizes the channel utilization in Wi-Fi hot spots when there are $M$ users, hereafter indicated as STAs, which use TCP connections to download traffic from the Internet. We consider only downstream data traffic because TCP-based downloads are the dominant part of the user traffic in typical local area networks [6]. The extension of our model to deal with upstream data traffic is left to future works. To the best of authors' knowledge this is the first attempt to model the interaction between the 802.11 MAC protocol and the TCP considering this increasingly important network architecture. The key approximation used in our model is the assumption that the devices access the wireless slotted channel adopting a $p$-persistent IEEE $802.11 \mathrm{~b}$ protocol $[5,7]$. This assumption is commonly used to study the binary exponential backoff adopted in the 802.11 MAC protocol [1]. However, our work significantly differs from previous studies because we integrate into the model the feedbackbased behavior of the TCP protocol. Specifically, one of the major consequences of the flow control techniques used by the TCP protocol is that the amount of acknowledgment traffic the STAs have to send back to the AP mainly depends on the rate the AP delivers the TCP data packets to the STAs. In [8] we show that, even with asymptotic TCP connections, the STAs may not have a TCP ACK immediately available for transmission. Our model takes into consideration that the instantaneous number of STAs participating to the channel contention is variable. By differentiating the AP's and STAs' traffic arrival processes, we can model the way the TCP feedback-based behavior affects the MAC protocol operations. Our model provides an in-depth understanding of the MAC protocol operations in hot spot networks. By exploiting our analytical results, in this paper we show that: 1 . even with a large number of TCP connections, on average there are less than two STAs contending with the AP for the channel bandwidth; 2. the channel utilization is almost independent of the number of downstream TCP connections; and 3. the MAC protocol efficiency depends mainly on the AP's capability to pump traffic towards the STAs. It is worth pointing out that observations 1.-3. are counter-intuitive results, because they indicate that the users' throughput is limited by the interaction of the MAC protocol with the TCP flow control mechanisms rather than the contention level of the network. 


\section{Model Definition}

In our study, we have considered a hot spot with a fixed number $M$ of users (the STAs) that are associated to the AP. In the analysis we assume ideal channel conditions (i.e., no hidden terminals or packet losses). The stations access the wireless channel adopting a $p$-persistent IEEE $802.11 \mathrm{~b}$ MAC protocol $[5,7]$, which differs from the standard protocol only in the backoff selection: instead of using the binary exponential backoff algorithm, the backoff interval is sampled from a geometric distribution of parameter $p$. Since the STAs and the AP can suffer different number of collisions, we assume that the AP and the STAs use different $p$ values: $p_{A P}$ for the AP, and $p_{S T A}$ for the STAs. Each station performs a TCP-based download from remote Internet servers. Hence, the AP delivers TCP data packets towards the STAs, while the STAs reply back with TCP ACKs. The TCP sources are asymptotic connections, i.e., they have always a packet ready to be transmitted (ftp-like traffic). We assume that the AP and the STAs have infinite size buffers, therefore neither TCP data packets nor TCP ACKs can be lost due to buffer overflows. The key approximation of our model is the $p$-persistent behavior of the MAC protocol. However, the dynamic of the TCP protocol is complex and to make the problem analytically tractable we have adopted further simplifying assumptions. Specifically, we assumed that each TCP data packet is acknowledged separately, that is the delayed-ACK mechanism [9] is disabled. This implies that the TCP receivers generate the maximum offered load for the network, and this is the worst-case situation for the TCP throughput performance and the contention on network resources. The last assumption we have made is to consider that no more than one TCP ACK is queued in the active STAs' buffers ${ }^{1}$. The major outcomes of this assumption are: 1) after a STA's successful transmission the STA's buffer is empty and the STA becomes inactive; and 2) each AP's successful transmission activates a new STA. As a consequence, during the analytical study we can assume that the number of active STAs in the network is a discrete random variable that increments by one every time the AP performs a successful transmission, and decrements by one every time a STA performs a successful transmission. Preliminary simulations results have substantiated this approximation $[8,10]$.

\section{Model Analysis}

In this section we outline the analytical study we have developed to compute the channel utilization. Due to the space constraints we present the main results and discuss the line of reasoning we followed. The complete mathematical development and the formal proofs are presented in the technical report [11], available at the url http://cnd.iit.cnr.it/tech-report/2003/. To model the MAC protocol behavior, we observe the channel between two consecutive successful transmissions. From the $p$-persistent behavior it is straightforward to derive that all the stochastic processes that define the occupancy pattern of the

\footnotetext{
${ }^{1}$ An active STA is a STA with a not empty transmission buffer.
} 


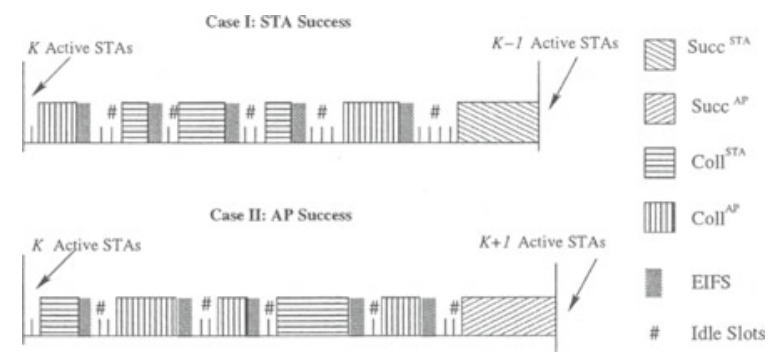

Fig. 1. Structure of channel events between to consecutive successful transmissions

channel (i.e., idle slots, collisions and successful transmissions) are regenerative, since they renew at the end of each successful transmission. In the following we will exploit the regenerative properties of the system and classical renewal theoretical arguments [12] to compute the time between two AP's successful transmissions, say $T_{v}$. Hereafter, we will refer to $T_{v}$ also as virtual transmission time. During the development of the analysis we assume to know the steady state probability that there are $K$ active STAs after the AP's successful transmissions, denoted as $\pi(K)$, with $K \leq M$. In section 3.1 we present approximated formulas to compute the $\pi(K)$. First of all, let us introduce the notation that will be useful in the following analysis:

- $E[X]_{K}$ is the expectation of the random variable $X$ conditioned to having $K$ active STAs;

- $I_{A P}$ is a function that has value 1 when the AP performs a transmission attempt, 0 otherwise;

- Idle $p$ is the duration of the idle time that precedes a transmission attempt;

- Coll and Succ are, respectively, the duration of a collision and a successful transmission (including all the MAC protocol overheads due to interframe spaces and control frames);

- given the random variable $X$, we define $X^{A P}=\left\{X, I_{A P}=1\right\}$ and $X^{S T A}=$ $\left\{X, I_{A P}=0\right\}$

- $t_{B}, t_{H}$ and $t_{A C K}$ are, respectively, the time needed to transmit a byte, the overhead added to the MAC data payload, and the MAC ACK frame;

- $t_{S L O T}$ and $\tau$ are, respectively, the time slot duration and the maximum propagation delay over the wireless channel.

To derive the closed formula of $E\left[T_{v}\right]_{K}$, i.e., the average virtual transmission time conditioned to having $K$ active STAs at the beginning of the $T_{v}$, it is useful to consider which events occur between two consecutive successful transmissions. Hereafter, we denote the time interval between two consecutive successful transmissions as $T_{s}$. Again, let us assume that the number of active STAs is $K$. In Fig. 1 we show the structure of the $T_{s}$. Collisions and idle periods may occur before a successful transmission. An idle period is a time interval in which the transmission medium remains idle due to the backoff algorithm. A collision may 
either involve the AP, or be caused only by STAs' concurrent transmission attempts. After the successful transmission, the hot spot contention level, that is the number of active STAs, changes. The analytical study is simplified by assuming that in the active STAs' buffers there is at most one queued TCP ACK. Hence, if an AP's successful transmission completes the $T_{s}$, the number of active STAs changes from $K$ to $K+1$, since the AP's transmission causes an inactive STAs to generate a TCP ACK. On the other hand, if a STA's successful transmission completes the $T_{s}$, the number of active STAs changes from $K$ to $K-1$, since the STA has sent the only TCP ACK it has. With reference to a generic $T_{s}, N_{c}^{A P}$ indicates the number of collisions that involve the AP, and $N_{c}^{S T A}$ indicates the number of collisions that don't involve the AP. Considering the system behavior we can write that

$$
\begin{aligned}
& E\left[T_{s} \mid S u c c^{A P}\right]_{K}=E\left[\sum_{i=1}^{N_{c}^{A P}}\left(I d l_{-} p_{i}+C_{\text {Coll }}^{A P}+\tau+E I F S\right)+\sum_{i=1}^{N_{c}^{S T A}}\left(I d l e_{-} p_{i}+\right.\right. \\
& \left.\left.\operatorname{Coll}_{i}^{S T A}+\tau+E I F S\right)\right]_{K}+E\left[\text { Idle } p_{N_{c}^{S T A}+N_{c}^{A P}+1}\right]_{K}+E\left[\operatorname{Succ}^{A P}\right]_{K}, \\
& E\left[T_{s} \mid S u c c^{S T A}\right]_{K}=E\left[\sum_{i=1}^{N_{c}^{A P}}\left(I d l e_{-} p_{i}+\text { Coll }_{i}^{A P}+\tau+E I F S\right)+\sum_{i=1}^{N_{c}^{S T A}}\left(I d l e_{-} p_{i}+\right.\right. \\
& \left.\left.\operatorname{Coll}_{i}^{S T A}+\tau+E I F S\right)\right]_{K}+E\left[\text { Idle_p }_{N_{c}^{S T A}+N_{c}^{A P}+1}\right]_{K}+E\left[\text { Succ }^{S T A}\right]_{K},
\end{aligned}
$$

where $T_{s} \mid S u c c^{S T A}$ is the Case I shown in Fig. 1, whereas $T_{s} \mid S u c c^{A P}$ is the Case II shown in Fig. 1. The assumption on the $p$-persistent behavior of the backoff algorithm implies that the idle periods $\left\{\right.$ Idle $\left.p_{i}\right\}$, and the collisions times $\left\{\mathrm{Coll}_{i}^{A P}, \mathrm{Coll}_{i}^{S T A}\right\}$ are i.i.d. random variables. Thus (1a) and (1b) can be rewritten as

$$
\begin{aligned}
E\left[T_{s} \mid S u c c^{A P}\right]_{K}=\left(E\left[N_{c}^{A P} \mid N_{t r} \geq 1\right]_{K}+E\left[N_{c}^{S T A} \mid N_{t r} \geq 1\right]_{K}+1\right) \cdot E\left[\text { Idle }_{-}\right]_{K}+ \\
\quad E\left[N_{c}^{A P} \mid N_{t r} \geq 1\right]_{K} \cdot\left(E\left[\text { Coll }^{A P}\right]_{K}+\tau+E I F S\right)+E\left[N_{c}^{S T A} \mid N_{t r} \geq 1\right]_{K} \cdot \\
\quad\left(E\left[\text { Coll }^{S T A}\right]_{K}+\tau+E I F S\right)+E\left[S u c c^{A P}\right]_{K}, \\
E\left[T_{s} \mid S u c c^{S T A}\right]_{K}=\left(E\left[N_{c}^{A P} \mid N_{t r} \geq 1\right]_{K}+E\left[N_{c}^{S T A} \mid N_{t r} \geq 1\right]_{K}+1\right) \cdot E\left[\text { Idle }_{-} p\right]_{K}+ \\
\quad E\left[N_{c}^{A P} \mid N_{t r} \geq 1\right]_{K} \cdot\left(E\left[\text { Coll }^{A P}\right]_{K}+\tau+E I F S\right)+E\left[N_{c}^{S T A} \mid N_{t r} \geq 1\right]_{K} \cdot \\
\left(E\left[\text { Coll }^{S T A}\right]_{K}+\tau+E I F S\right)+E\left[S u c c^{S T A}\right]_{K} .
\end{aligned}
$$

The average number of collisions that involve the AP during a $T_{s}$ can be further expanded in

$$
E\left[N_{c}^{A P} \mid N_{t r} \geq 1\right]_{K}=E\left[N_{c}^{A P} \mid I_{A P}=1, N_{t r} \geq 1\right]_{K} \cdot \operatorname{Pr}\left\{I_{A P}=1 \mid N_{t r} \geq 1\right\}_{K},
$$

where $\operatorname{Pr}\left\{I_{A P}=1 \mid N_{t r} \geq 1\right\}_{K}$ is the probability that the AP transmits given that there is at least a transmission on the channel and there are $K$ active STAs. Similarly, denoting with $\operatorname{Pr}\left\{I_{A P}=0 \mid N_{t r} \geq 1\right\}_{K}$ the probability that the AP isn't transmitting, given that there is at least a transmission on the channel and there 
are $K$ active STAs, the average number of collisions that don't involve the AP during a $T_{s}$ can be further expanded in

$$
E\left[N_{c}^{S T A} \mid N_{t r} \geq 1\right]_{K}=E\left[N_{c}^{S T A} \mid I_{A P}=0, N_{t r} \geq 1\right]_{K} \cdot \operatorname{Pr}\left\{I_{A P}=0 \mid N_{t r} \geq 1\right\}_{K} .
$$

The following Lemma provides closed formulas for the expressions in (2a) and (2b).

Lemma 1. By assuming that: $i)$ the STAs sample the backoff interval from a geometric distribution of parameter $p_{S T A}$, while the AP samples the backoff interval from a geometric distribution of parameter $p_{A P}$; ii) the number of active STAs at the beginning of the $T_{s}$ is $K$; and iii) the sequence of message lengths $\left\{l_{i}^{A P}\right\}\left(\left\{l_{i}^{A P}\right\}\right)$ of AP's transmissions (STAs' transmissions), expressed in bytes, are i.i.d. random variables with mean $\left.\bar{l}_{A P} \bar{l}_{S T A}\right)$, it holds that

$$
\begin{aligned}
& \operatorname{Pr}\left\{I_{A P}=0 \mid N_{t r} \geq 1\right\}_{K}=\sum_{n=1}^{K} \frac{\left(\begin{array}{c}
K \\
n
\end{array}\right)\left(1-p_{A P}\right) \cdot p_{S T A}^{n}\left(1-p_{S T A}\right)^{K-n}}{1-\left(1-p_{A P}\right)\left(1-p_{S T A}\right)^{K}} \\
& \operatorname{Pr}\left\{I_{A P}=1 \mid N_{t r} \geq 1\right\}_{K}=\sum_{n=0}^{K} \frac{\left(\begin{array}{c}
K \\
n-1
\end{array}\right) p_{A P} \cdot p_{S T A}^{n}\left(1-p_{S T A}\right)^{K-n}}{1-\left(1-p_{A P}\right)\left(1-p_{S T A}\right)^{K}} \\
& E\left[I d l_{-} p\right]_{K}=\frac{\left(1-p_{A P}\right)\left(1-p_{S T A}\right)^{K}}{1-\left(1-p_{A P}\right)\left(1-p_{S T A}\right)^{K}} \cdot t_{S L O T} \\
& E\left[N_{c}^{A P} \mid I_{A P}=1, N_{t r} \geq 1\right]_{K}=\frac{1-\left(1-p_{S T A}\right)^{K}}{\left(1-p_{S T A}\right)^{K}} \\
& E\left[\left.N_{c}^{S T A}\right|_{A P}=0, N_{t r} \geq 1\right]_{K}=\frac{1-\left[\left(1-p_{S T A}\right)^{K}+K p_{S T A}\left(1-p_{S T A}\right)^{K-1}\right]}{K p_{S T A}\left(1-p_{S T A}\right)^{K-1}} \\
& E\left[\operatorname{Coll}^{A P}\right]_{K}=t_{H}+\frac{t_{B}}{1-\left(1-p_{S T A}\right)^{K}} \sum_{m=1}^{l_{M A X}} m\left\{F _ { A P } ( m ) \left\{1-p_{S T A}\right.\right. \\
& \left.\quad\left[1-F_{S T A}(m)\right]\right\}^{K}-F_{A P}(m-1)\left\{1-p_{S T A}\left[1-F_{S T A}(m-1)\right]\right\}^{K}- \\
& \left.\left[F_{A P}(m)-F_{A P}(m-1)\right]\left(1-p_{S T A}\right)^{K}\right\} \\
& E\left[\operatorname{Coll}^{S T A}\right]_{K}=t_{H}+\frac{t_{B}}{1-\left[\left(1-p_{S T A}\right)^{K}+K p_{S T A}\left(1-p_{S T A}\right)^{K-1}\right]} \\
& \sum_{M A X} m\left\{\left\{1-p_{S T A}\left[1-F_{S T A}(m)\right]\right\}^{K}-\left\{1-p_{S T A}\left[1-F_{S T A}(m-1)\right]\right\}^{K}\right. \\
& \sum_{m=1} m \\
& \left.-\left[F_{S T A}(m)-F_{S T A}(m-1)\right] p_{S T A}\left(1-p_{S T A}\right)^{K-1}\right\} \\
& E\left[S u c c^{A P}\right]_{K} \leq 2 \tau+t_{H}+t_{B} \cdot \bar{l}_{A P}+S I F S+t_{A C K}+D I F S \\
& E\left[S u c c^{S T A}\right]_{K} \leq 2 \tau+t_{H}+t_{B} \cdot \bar{l}_{S T A}+S I F S+t_{A C K}+D I F S
\end{aligned}
$$

where $F_{A P}(m)=\operatorname{Pr}\left\{l_{A P} \leq m\right\}$ and $F_{S T A}(m)=\operatorname{Pr}\left\{l_{S T A} \leq m\right\}$.

Proof. See Appendix I in [11]. 
Using the $E\left[T_{s} \mid S u c c^{A P}\right]_{K}$ and $E\left[T_{s} \mid S u c c^{S T A}\right]_{K}$ formulas, we are able to express the $E\left[T_{v}\right]_{K}$. The following Lemma defines a recursive algorithm to compute the $E\left[T_{v}\right]_{K}$.

Lemma 2. By assuming that: $i$ ) the devices access the channel according to a p-persistent access scheme; ii) the number of active STAs at the beginning of the $T_{v}$ is $K$; and iii) there is at most one ACK queued in the active STAs' buffers, it holds that

$$
\left\{\begin{aligned}
E\left[T_{v}\right]_{K}= & \left\{E\left[T_{s} \mid S u c c^{S T A}\right]_{K}+E\left[T_{v}\right]_{K-1}\right\} \cdot \operatorname{Pr}\left\{S u c c^{S T A} \mid S u c c\right\}_{K}+ \\
& E\left[T_{s} \mid S u c c^{A P}\right]_{K} \cdot \operatorname{Pr}\left\{S u c c^{A P} \mid S u c c\right\}_{K} \\
E\left[T_{v}\right]_{0}= & E[\text { Idle_p }]_{0}+E\left[S u c c^{A P} \mid S u c c\right]_{0}
\end{aligned}\right.
$$

where $\operatorname{Pr}\left\{S u c c^{S T A} \mid S u c c\right\}_{K}$ and the $\operatorname{Pr}\left\{S u c c^{A P} \mid S u c c\right\}_{K}$, are, respectively, the probability that a successful transmission belongs to either the Case I of Fig. 1 or Case II of Fig. 1.

Proof. In [11].

Finally, the $E\left[T_{v}\right]$ value is computed as

$$
E\left[T_{v}\right]=\sum_{K=1}^{M} E\left[T_{v}\right]_{K} \cdot \pi(K)
$$

By exploiting the analytical formulas we have derived so far, it is straightforward to compute the average channel utilization achieved by the AP's transmissions, say $\rho_{A P}$. In fact, $\rho_{A P}$ is the ratio between the average time the channel is occupied by the payload transmission of the AP's successful transmission and the average virtual transmission time, that is

$$
\rho_{A P}=\frac{t_{B} \cdot \bar{l}_{A P}}{E\left[T_{v}\right]} .
$$

To derive the average channel utilization achieved by the STAs' transmissions, say $\rho_{S T A}$, we have to calculate the average number of STAs' successful transmissions, say $E\left[N_{s u c c}^{S T A}\right]$, which occur during a $T_{v}$. Specifically, $\rho_{S T A}$ is the ratio between the average time the channel is occupied by the payload transmission of the STAs' successful transmissions and the average virtual time. Similarly to the reasoning used in Lemma 2, we start by deriving the $E\left[N_{\text {succ }}^{S T A}\right]_{K}$, i.e., the average number of STAs' successful transmissions during a $T_{v}$, conditioned to having $K$ active STAs at the beginning of the $T_{v}$. The following Lemma provides a recursive algorithm to compute $E\left[N_{\text {succ }}^{S T A}\right]_{K}$

Lemma 3. By assuming that: $i$ ) the devices access the channel according to a p-persistent access scheme; ii) the number of active STAs at the beginning of the $T_{v}$ is $K$; and iii) there is at most one TCP ACK queued in the active STAs' buffers, it holds that

$$
\left\{\begin{array}{l}
E\left[N_{\text {succ }}^{S T A}\right]_{K}=\left\{1+E\left[N_{\text {succ }}^{S T A}\right]_{K-1}\right\} \cdot \operatorname{Pr}\left\{S u c c{ }^{S T A} \mid S u c c\right\}_{K} \\
E\left[N_{\text {succ }}^{S T A}\right]_{0}=0
\end{array}\right.
$$


Proof. In [11].

Similarly to (4), it follows that $E\left[N_{\text {succ }}^{S T A}\right]=\sum_{K=1}^{M} E\left[N_{\text {succ }}^{S T A}\right]_{K} \cdot \pi(K)$. Hence, it holds that

$$
\rho_{S T A}=\frac{t_{B} \cdot \bar{l}_{S T A} \cdot E\left[N_{\text {succ }}^{S T A}\right]}{E\left[T_{v}\right]} .
$$

Finally, the overall channel utilization, say $\rho$, is computed as

$$
\rho=\rho_{A P}+\rho_{S T A}
$$

The maximum achievable $\rho$ identifies the MAC protocol capacity, which provides a good indication of the overheads introduced by the MAC protocol to perform its coordination task among devices. The derivation of the couple of $\left\{p_{A P}, p_{S T A}\right\}$ values that guarantees to attain the protocol capacity is left to further studies.

\subsection{Estimation of $\pi(K)$}

The analytical study we have developed so far requires the knowledge of the probability that there are $K$ active STAs after an AP's successful transmission, denoted as $\pi(K)$. In this section we propose a simple model to derive the $\pi(K)$. It is worth pointing out that the analytical framework presented in the previous section is independent of the $\pi(K)$ formula. However, an accurate estimation of the $\pi(K)$ improves also the accuracy of the $\rho$ computation. The $p$-persistent behavior of AP and STAs implies that each transmission attempt, regardless of the number of consecutive collisions experienced by the AP or the STAs, is a successful transmission with a constant and independent probability (see Lemma 1). Therefore, the stochastic process that describes the number of active STAs after an AP's successful transmission, say $S(n)$, can be modeled through an embedded Markov process. The time scale adopted is discrete: the index $n$ refers to the beginning of the $n^{t h}$ virtual transmission time $T_{v}$. The probability $\pi(K)$, for $K=1,2, \ldots, M$, is the steady state probability of the chain, that is $\pi(K)=\lim _{n \rightarrow \infty} \operatorname{Pr}\{S(n)=K\}$. In Fig. 2 we depict the Markov chain that models the $S(n)$ process when the $M$ value is finite. Henceforth, to indicate the transition probability from state $i$ to state $j$, i.e., the $\operatorname{Pr}\{S(n+1)=j \mid S(n)=i\}$, we use the notation $p(i, j)$. The following Lemma derives the $p(i, j)$ for the chain depicted in Fig. 2.

Lemma 4. Under the assumption that: $i)$ the AP and the STAs have the same probability to perform a successful transmission; and ii) no more than one TCP $A C K$ is queued in the active STAs' buffers, it holds that

$$
\begin{cases}p\left(K_{1}, K_{0}\right)=\frac{1}{K_{0}+1} & K_{0}=1,2, \ldots, M-1 \\ & K_{1}=1,2, \ldots, K_{0}+1 \\ p\left(K_{1}, M\right)=\frac{1}{M+1} & K_{1}=1,2, \ldots, M-1 \\ p(M, M)=\frac{2}{M+1} & \end{cases}
$$




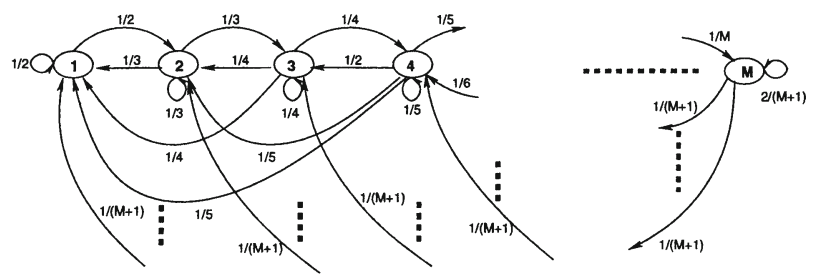

Fig. 2. Markov chain model of the $S(n)$ process for a finite $M$.

Proof. In [11].

Using the knowledge of the transition probabilities $p(i, j)$ given in (8), it is straightforward to find a closed formula for the $\pi(K)$ by writing the equilibrium at each node of the chain. Thus all the $\pi(K)$ are expressed in terms of $\pi(1)$ and of the $K$ value. Finally, $\pi(1)$ is computed by imposing the normalization condition on the steady state probabilities (see [11] for the details). The $E\left[N_{S T A}\right]$ can be computed as $E\left[N_{S T A}\right]=\sum_{K=1}^{M} K \cdot \pi(K)$. In [11] we proved that $E\left[N_{S T A}\right] \leq 2$. Thus, in hot spot networks the contention level is very low. In [11] it is considered also the case of an infinite number of STAs, that is $M \rightarrow \infty$, and it is proved the following proposition.

Proposition 1. When $M \rightarrow \infty$, the average number of active STAs that are active at the beginning of $a T_{v}$ is $E\left[N_{S T A}\right]=\sum_{K=1}^{\infty} K \cdot \pi(K)=\sum_{K=1}^{\infty} \frac{K}{e(K-1) !}=$ 2 .

This result is counter-intuitive because it demonstrates that, even when there are an infinite number of asymptotic TCP connections, the contention level in the network is very low. On average, only two STAs contend with the AP for the channel bandwidth. In [11] numerical results confirming the accuracy of the proposed $\pi(K)$ estimation are shown.

\section{Model Validation}

In this section we validate the correctness and accuracy of the analytical study performed in section 3 by means of realistic simulations. The simulation tool we have used is an extension of the one used in [5] and [7]. In addition to all the IEEE 802.11b MAC standard protocol details, it implements also the behavior of asymptotic TCP connections. The TCP version we have used is the TCP Reno [9] with the delayed-ACK mechanism disabled. The formulas we have derived in section 2 are applicable to a general payload distribution. In the following study we consider a constant distribution for the payload length. If not otherwise specified, the TCP senders delivers TCP data packets with a payload equal to 1500 bytes. As a consequence, we used $\bar{l}_{A P}=1540$ bytes and $\bar{l}_{S T A}=40$ bytes $^{2}$ to compute the numerical results. As far as the $p_{A P}$

\footnotetext{
${ }^{2}$ The $\bar{l}_{A P}$ value characterizes a TCP data packet with payload equal to 1500 bytes, while the $\bar{l}_{S T A}$ value is the typical size of a TCP ACK.
} 


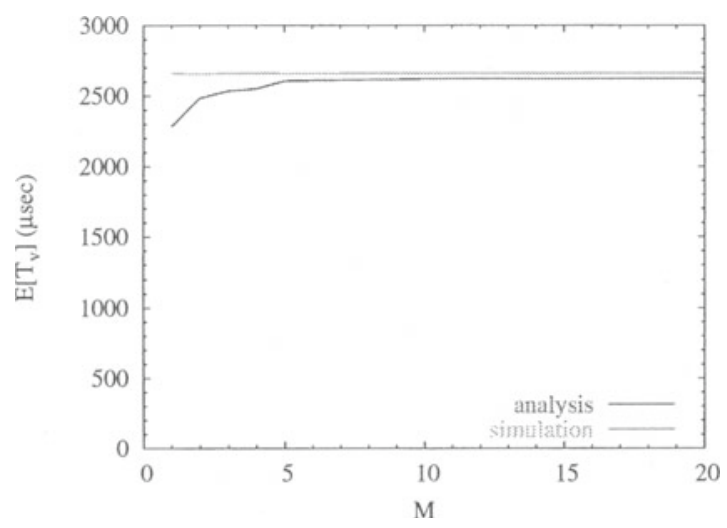

Fig. 3. $E\left[T_{v}\right]$ : analysis versus simulation

and $p_{S T A}$ values, we use the values estimated through the iterative algorithm described in Appendix II of [11]. The constant overheads of the IEEE 802.11 MAC protocol are fully compliant with the standard and the values we used are the same adopted in [7]. All the results presented henceforth were obtained by performing simulation runs long enough to guaranteed a $99 \%$ confidence level with a precision lower that $1 \%$. The first set of simulations was run to validate the accuracy of formula (4). In Fig. 3 we plot the average virtual time versus the number $M$ of users, with $M \in[1, \ldots, 20]$, and we compare the analytical results against the behavior of the real system. Fig. 3 confirms that the $p$-persistent model provides a close approximation of the real behavior at least for $M \geq 5$. The analytical results are slightly lower than the simulation results for $M<5$ because assuming that the active STAs have at most a TCP ACK to transmit is less accurate with a few stations. However, despite of this slight difference our model accurately captures the dynamic of the contention level in the hot spot network. Lemma 2 shows that the $E\left[T_{v}\right]$ depends on the $E\left[T_{v}\right]_{K}$ distribution. For this reason, we have not limited our study to the $E\left[T_{v}\right]$ computation, but we have also investigated if our model is able to match the $E\left[T_{v}\right]_{K}$ distribution. Fig. 4(a) and Fig. 4(b) show the $E\left[T_{v}\right]_{K}$ respectively for $M=5$ and $M=20$. These figures indicate that the analytical results are slightly lower than the simulation results, with an error that never exceeds the $10 \%$. In Fig. 5 we show the channel utilization $\rho$ for several $M$ values, $M \in[1 \ldots 20]$. The numerical results show a close correspondence between the analysis and the simulation for $M \geq 5$. The differences when $M<5$ are due to the underestimate introduced by our model in the $E\left[T_{v}\right]$ computation for small $M$ values. Another important outcome of our analysis is that the channel utilization is almost independent on the number $M$ of stations (see Fig. 5). This can be explained by considering the TCP feedbackbased behavior. Specifically, the more data traffic the AP sends to the STAs, the more STAs become active. However, the larger is the number of active STAs the lower is the probability that the AP can perform a successful transmission 


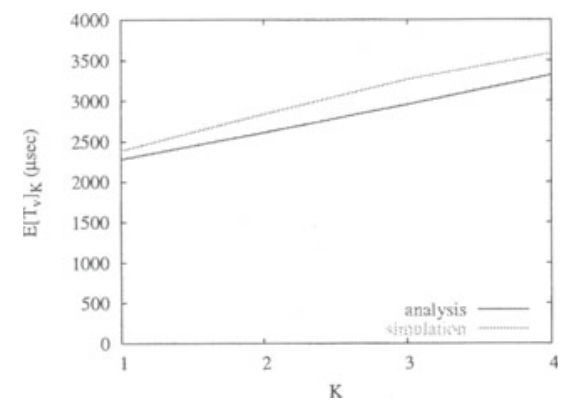

(a) $M=5$

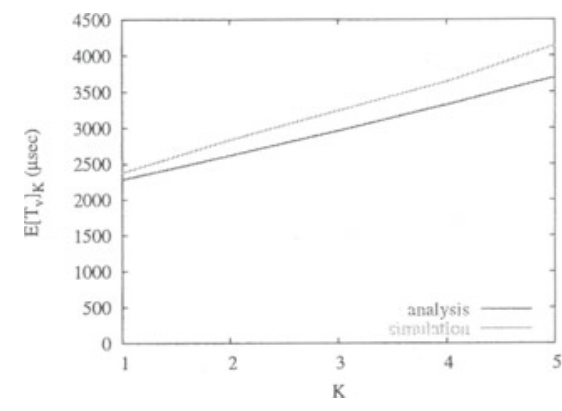

(b) $M=20$

Fig. 4. $E\left[T_{v}\right]_{K}$ for various $M$ : analysis versus simulation

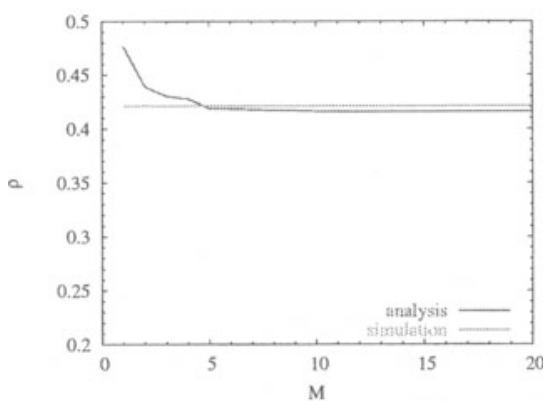

Fig. 5. $\rho$ : analysis versus simulation

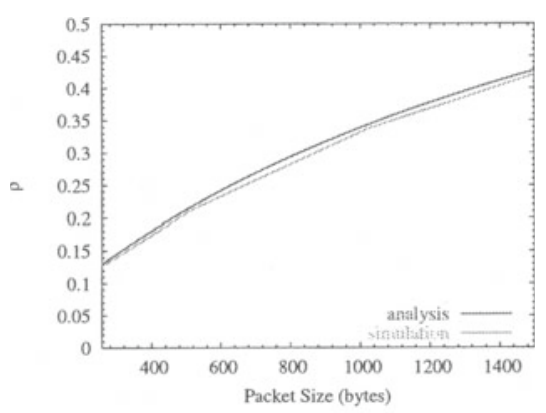

Fig. 6. $\rho$ for different payload length and $M=10$ : analysis versus simulation

to activate new stations. Therefore the contention level in the network cannot increase by increasing the number $M$ of users. A last set of simulations was run to investigate the impact of the payload length over both the throughput performance and the model accuracy. In Fig. 6 we show the channel utilization in a network with $M=10$ by varying the $\bar{l}_{A P}$ value. Specifically, Fig. 6 shows the channel utilization $\rho$ versus $\bar{l}_{A P} \in[256, \ldots, 1540]$ bytes, with $\bar{l}_{S T A}=40$ bytes as in the previous experiments. As expected $\rho$ increases as the payload size increases because the fraction of channel time per successful transmission that is occupied by the protocol overheads reduces. The numerical results clearly confirm that the model accuracy is independent of the payload length.

\section{Conclusions}

To the best of authors' knowledge, this work is the first attempt of developing an analytical model to compute the channel utilization in hot spot networks. 
Our model defines the contention level of the network as the number of STAs with a packet available for transmission. By considering $i$ ) the feedback-based behavior of the TCP protocol, and ii) the fact that the STAs' traffic has to be delivered through the AP, we were able to model how the contention level changes. This allowed us to derive an accurate characterization of the MAC protocol operations, with a good correspondence between the analytical results and the simulation results. By exploiting the developed analysis, we provided thorough reasons of the following counter-intuitive observations: $i$ ) the channel utilization is almost independent of the number of TCP connections; ii) on average the access point contends with few users for the channel bandwidth. This is significantly different from the results of previous analytical studies that considered UDP-like traffic. In fact, with asymptotic not-responsive traffic flows the channel utilization degrades as the number of users increases.

\section{References}

1. Part 11: Wireless LAN Medium Access Control (MAC) and Physical Layer (PHY) Specification/Amendment 2: Higher-speed Physical Layer (PHY) in the 2:4 GHz band (2001)

2. Kleinrock, L., Tobagi, F.: Packet Switching in Radio Channels: Part I - Carrier Sense Multiple-Access Modes and their Throughput-Delay Characteristics. IEEE Trans. Commun. 23 (1975) 1400-1416

3. Chhaya, H., Gupta, S.: Performance modeling of asynchronous data transfer methods on IEEE 802.11 MAC protocol. Wireless Networks 3 (1997) 217-234

4. Bianchi, G.: Performacs Analysis of the IEEE 802.11 Distributed Coordination Function. IEEE J. Select. Areas Commun. 18 (2000) 1787-1800

5. Calí, F., Conti, M., Gregori, E.: Dynamic Tuning of the IEEE 802.11 Protocol to Achieve a Theoretical Throughput Limit. IEEE/ACM Trans. Networking 8 (2000) 785-799

6. Tang, D., Baker, M.: Analysis of a Local-Area Wireless Network. In: Proc. of MobiCom 2000, Boston, MA (2000)

7. Bruno, R., Conti, M., Gregori, E.: Optimization of Efficiency and Energy Consumption in p-Persistent CSMA-Based Wireless LANs. IEEE Trans. Mob. Comp. 1 (2002) 10-31

8. Bruno, R., Conti, M., Gregori, G.: Throughput Evaluation and Enhancement of TCP clients in Wi-Fi Hot Spots. In: Proc. of WONS 2004, Madonna di Campiglio, Italy (2004) 73-86

9. Stevens, W.: TCP Illustrated, Volume 1: The Protocols. Addison-Wesley, New York, NY (2001)

10. Pilosof, S., Ramjee, R., Raz, D., Shavitt, Y., Sinha, P.: Understanding TCP fairness over wireless LAN. In: Proc. of IEEE Infocom 2003, San Francisco, CA (2003)

11. Bruno, R., Conti, M., Gregori, E.: Throughput Analysis of TCP Clients in Wi-Fi Hot Spot Networks. Technical report, IIT - CNR Pisa (2003)

12. Heyman, D., Sobel, M.: Stochastic Models in Operations Research. Volume 1. McGraw-Hill, New York, NY (1982) 